\title{
Strain longitudinal global: un parámetro útil para evaluar disfunción ventricular izquierda subclínica en el síndrome metabólico
}

\author{
Wilson Cañón-Montañez ${ }^{\mathrm{a}, \mathrm{b}, *}$, Ángela B.S. Santos ${ }^{\mathrm{b}}$ y Murilo Foppa ${ }^{\mathrm{b}, \mathrm{c}}$
}

\begin{abstract}
a Programa de Pós-Graduação em Epidemiologia, Faculdade de Medicina, Universidade Federal do Rio Grande do Sul (UFRGS), Porto Alegre, Brasil

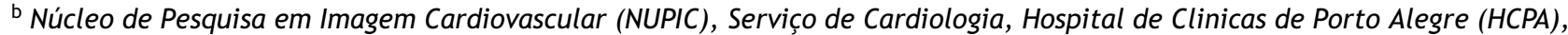
Porto Alegre, Brasil

c Programa de Pós-Graduação em Cardiologia, Faculdade de Medicina, Universidade Federal do Rio Grande do Sul (UFRGS), Porto Alegre, Brasil
\end{abstract}

Recibido el 20 de agosto de 2015; aceptado el 1 de octubre de 2015

Disponible en Internet el 23 de diciembre de 2015

\author{
PALABRAS CLAVE \\ Síndrome metabólico; \\ Función ventricular \\ izquierda; \\ Disfunción diastólica; \\ Contracción \\ miocárdica
}

\begin{abstract}
Resumen La alta prevalencia del síndrome metabólico es un fenómeno global. Debido a su aumento concomitante con el riesgo de desarrollar diabetes mellitus y enfermedad cardiovascular el síndrome metabólico tendrá una enorme carga sobre las economías del mundo y en la salud pública si no se producen intervenciones precoces y eficaces. Uno de los mecanismos propuestos para aumentar el riesgo cardiovascular se relaciona con la disfunción sistólica y diastólica subclínica encontrada en estos pacientes. La fracción de eyección, el parámetro más utilizado para la evaluación de la función sistólica, tiene baja sensibilidad para la valoración de disfunción temprana de la contractilidad miocárdica. El desarrollo de nuevas técnicas ecocardiográficas, como strain longitudinal global por ecocardiografía bidimensional speckle-tracking ha reforzado la capacidad de evaluar la función del ventrículo izquierdo mediante evaluación cuantitativa de la deformación miocárdica. La detección y el manejo precoz de la enfermedad cardiovascular subclínica puede mitigar algunos de los riesgos cardiovasculares asociados con trastornos metabólicos. Con capacidad de detectar disfunción ventricular izquierda subclínica en pacientes con síndrome metabólico, el strain longitudinal global medido por ecocardiografía bidimensional speckle-tracking tiene el potencial de convertirse en una herramienta útil para la estratificación del riesgo en esta población.

(c) 2015 Sociedad Colombiana de Cardiología y Cirugía Cardiovascular. Publicado por Elsevier España, S.L.U. Este es un artículo Open Access bajo la licencia CC BY-NC-ND (http://creativecommons.org/licenses/by-nc-nd/4.0/).
\end{abstract}

\footnotetext{
* Autor para correspondencia.

Correo electrónico: wilcamo32@yahoo.com (W. Cañón-Montañez).
} 


\section{KEYWORDS}

Metabolic syndrome; left ventricular function; diastolic function; myocardial contraction

\section{Global longitudinal strain: a useful parameter to assess subclinical left ventricular dysfunction in metabolic syndrome}

\begin{abstract}
The high prevalence of metabolic syndrome is a global phenomenon. Due to the increase of the concomitant risk of developing diabetes mellitus and cardiovascular diseases, metabolic syndrome will place a great burden on world economies and public health unless early and efficient action interventions are conducted. One of the suggested mechanisms in order to increase the cardiovascular risk is related to subclinical systolic and diastolic dysfunction discovered in these patients. Ejection fraction, the most frequently used parameter to assess systolic function, presents a low sensitivity to the assessment of early dysfunction of myocardial contractility. The development of new echocardiographic techniques, such as global longitudinal strain by two-dimensional speckle-tracking echocardiography have strengthened the ability to assess left ventricle function with a quantitative assessment of myocardial deformation. Early detection and management of subclinical cardiovascular diseases can mitigate some of the cardiovascular risks associated to metabolic disorders. With its ability to detect subclinical left ventricular dysfunction in patients with metabolic syndrome, global longitudinal strain measured with two-dimensional speckle-tracking echocardiography has the potential to become a useful tool for risk stratification in this population group.

(c) 2015 Sociedad Colombiana de Cardiología y Cirugía Cardiovascular. Published by Elsevier España, S.L.U. This is an open access article under the CC BY-NC-ND license (http://creativecommons.org/licenses/by-nc-nd/4.0/).
\end{abstract}

\section{Introducción}

Las enfermedades cardiovasculares son la principal causa de muerte en todo el mundo ${ }^{1}$. Recientemente, el estudio Global Burden of Disease 2013, estimó que casi el 30\% de todas las muertes en el mundo fueron causadas por estas ${ }^{1}$. Respecto a factores de riesgo para enfermedades cardiovasculares, existe evidencia que muestra que nueve factores de riesgo medidos fácilmente (tabaquismo, lípidos, hipertensión arterial, diabetes, obesidad, dieta, actividad física, consumo de alcohol y factores psicosociales) son responsables de más del $90 \%$ del riesgo de infarto agudo de miocardio en el mundo ${ }^{2}$.

El síndrome metabólico es un conjunto de factores de riesgo cardiometabólicos, que incluyen: obesidad abdominal, resistencia a la insulina, dislipidemia e hipertensión ${ }^{3}$. El agrupamiento de varios factores de riesgo para enfermedades cardiovasculares, como alteraciones en la glucosa y metabolismo de la insulina, obesidad abdominal, hipertensión arterial y dislipidemia han sido un asunto de fuerte debate desde la introducción del concepto de síndrome metabólico ${ }^{3}$.

En la mayoría de las personas con intolerancia a la glucosa o con diabetes mellitus tipo 2 , hay un conjunto de factores de riesgo que comúnmente aparecen juntos, formando lo que ahora se conoce como síndrome metabólico ${ }^{4}$. Las personas con este síndrome tienen un incremento del riesgo de desarrollar diabetes mellitus y enfermedades cardiovasculares, así como un riesgo adicional y substancial de desarrollo de eventos cardiovasculares clínicamente relevantes como infarto agudo de miocardio, accidente cerebrovascular y mortalidad por enfermedades cardiovasculares y por todas las causas ${ }^{5-7}$.

En relación con desenlaces cardiovasculares sustitutos o intermedios, el síndrome metabólico ha sido asociado con hipertrofia del ventrículo izquierdo y dilatación auricular ${ }^{8-10}$. El síndrome metabólico y la diabetes mellitus tipo 2 se relacionan con disfunción miocárdica identificada por imagen de doppler tisular (TDI) y ecocardiografía bidimensional speckle-tracking (2D-STE) ${ }^{11,12}$.

La 2D-STE aventaja al TDI de ángulo independencia, ya que ofrece la posibilidad de examinar no sólo la función longitudinal, sino un componente mayor del desempeño del ventrículo izquierdo ${ }^{13}$.

El desarrollo de nuevas técnicas ecocardiográficas, como la 2D-STE ha reforzado la capacidad de evaluar la función del ventrículo izquierdo mediante la evaluación cuantitativa de la deformación miocárdica ${ }^{14,15}$.

El Speckle-tracking evalúa la contractilidad del ventrículo izquierdo. Consiste en la captura y rastreo de puntos del ecocardiograma bidimensional a lo largo del ciclo cardiaco, generando vectores de movimiento y curvas de deformación (strain y strain rate). La deformación medida en planos ortogonales se denomina strain longitudinal, radial y circunferencial ${ }^{16}$.

Recientemente, algunos estudios, han mostrado que el strain longitudinal global (GLS) evaluado por 2D-STE, está reducido en sujetos con síndrome metabólico y fracción de eyección del ventrículo izquierdo (FEVI) normal, independiente de otros factores de riesgo cardiovascular ${ }^{17-19}$. Estos datos son útiles pues la identificación precoz de enfermedades cardiovasculares subclínicas puede ayudar a identificar a los individuos con mayor riesgo y mejorar los resultados clínicos en el síndrome metabólico ${ }^{20,21}$.

\section{Epidemiología y criterios diagnósticos del síndrome metabólico}

Se estima que alrededor del 20 al $25 \%$ de la población adulta del mundo, tiene síndrome metabólico. Por separado, los componentes del síndrome metabólico aumentan 
el riesgo de diabetes mellitus, enfermedades cardiovasculares y mortalidad por todas las causas ${ }^{22-24}$. La prevalencia relativamente alta del síndrome metabólico es un fenómeno global ${ }^{25,26}$, y esta parece estar aumentando debido a un crecimiento paralelo de la prevalencia de obesidad. La probabilidad de un incremento adicional del síndrome metabólico puede ser anticipada por causa de proyecciones de una mayor prevalencia de obesidad en el futuro ${ }^{27}$. En relación con datos de incidencia del síndrome metabólico, la literatura muestra pocos estudios longitudinales para determinarla. Algunos de ellos fueron realizados en Estados Unidos, Europa y Asia Oriental. En general, la incidencia reportada en estos estudios varía de $11 \%$ a $39 \%{ }^{28-31}$.

Varias instituciones en el mundo han propuesto criterios para el diagnóstico del síndrome metabólico. Entre ellas se destacan: Organización Mundial de la Salud (OMS), National Cholesterol Education Program Adult Treatment Panel III (NCEP-ATP III), International Diabetes Federation (IDF), Association of American Clinical Endocrinologists (AACE), European Group for the Study of Insulin Resistance (EGIR) y American Heart Association/National Heart, Lung and Blood Institute (AHA/NHLBI) ${ }^{32}$.

Recientemente, algunas organizaciones internacionales establecieron un consenso para la definición de síndrome metabólico ${ }^{33}$. Según este consenso el diagnóstico del síndrome requiere la presencia de al menos tres de los siguientes criterios: glicemia en ayunas (FPG) $\geq$ $100 \mathrm{mg} / \mathrm{dl}$; triglicéridos $\geq 150 \mathrm{mg} / \mathrm{dl}(1,7 \mathrm{mmol} / \mathrm{l})$; colesterol $\mathrm{HDL}<40 \mathrm{mg} / \mathrm{dl}(1,0 \mathrm{mmol} / \mathrm{l})$ en hombres $\mathrm{y}<50 \mathrm{mg} / \mathrm{dl}$ (1,3 mmol/l) en mujeres; obesidad abdominal [circunferencia de la cintura $\geq 94 \mathrm{~cm}$ en hombres $\mathrm{y} \geq 80 \mathrm{~cm}$ en mujeres], y presión arterial $\geq 130 / 85 \mathrm{~mm} \mathrm{Hg}$ o en tratamiento para hipertensión arterial.

\section{Ecocardiografía bidimensional speckle-tracking (2D-STE) - contractilidad y función miocárdica}

Los parámetros ecocardiográficos convencionales para la evaluación de la función sistólica presentan baja sensibilidad para la valoración exhaustiva de la contractilidad miocárdica. No detectan pequeños cambios de contractilidad o alteraciones precoces ${ }^{16}$.

La 2D-STE surgió recientemente como una nueva técnica y metodología desarrollada para el análisis de deformación miocárdica mediante el seguimiento automático del movimiento de patrones de puntos (speckles) inherentes a la interfaz de ultrasonido-miocardio ${ }^{34}$.

Recientemente, dos organizaciones internacionales: European Association of Cardiovascular Imaging (EACVI) y American Society of Echocardiography (ASE) publicaron un documento de consenso para estandarizar las técnicas de análisis de deformación miocárdica usando 2D-STE. El objetivo principal de esta guía es proporcionar las bases teóricas para explicar el significado fisiológico y el cálculo matemático de distintos parámetros, a los clínicos interesados en el uso de imágenes de deformación miocárdica para investigación y utilidad clínica, con la finalidad de asegurar un conocimiento común para las diferentes aplicaciones de esta técnica ecocardiográfica ${ }^{35}$.
Speckle-tracking, literalmente significa seguimiento de puntos o huellas y puede ser definido como el rastreo de puntos que forman la imagen bidimensional. Estos puntos también se denominan marcas acústicas digitales. Cada marca digital es formada por un pequeño conjunto de imágenes en escala gris, cuya disposición es única y caracteriza una porción particular del miocardio denominada patrón de seguimiento (speckle pattern) ${ }^{16}$. Esos patrones de puntos únicos como una "impresión digital" en el miocardio, son identificados y seguidos durante todo el ciclo cardíaco (fig. 1). Se generan vectores representativos del movimiento multidireccional, con sus respectivos valores y curvas graficadas en función del tiempo para varios parámetros: desplazamiento, velocidad de desplazamiento, strain y strain rate (figs. 2 y 3). Todos estos análisis integrados conforman la llamada dinámica de contracción del ventrículo izquierdo (Anexo,vídeo del material suplementario), una forma mucho más completa y sensible para caracterizar la función sistólica ${ }^{16,20}$.

\section{Conceptos claves del 2D-STE y strain longitudinal global}

Strain (deformación) y strain rate (velocidad de deformación) son índices clínicos de deformación miocárdica regional y global ${ }^{20}$. Ambos fueron introducidos y validados utilizando la imagen de resonancia magnética cardiaca y sonomicrometría (en modelos experimentales) como patrones de referencia ${ }^{36}$.

El strain (S) se define como el cambio fraccional en longitud de un segmento del miocardio, normalmente relacionado con la longitud al final de la diástole. El strain generalmente se expresa en porcentaje (\%). Si se representa el desplazamiento de las marcas acústicas en función del tiempo, se tendrá una curva de deformación de este punto de la pared, en función del tiempo. Esta curva se denomina strain rate $(\mathrm{SR})$ o velocidad o tasa de deformación y se expresa en $s$ - 1 o $1 / s^{16,20}$.

El strain y la strain rate pueden ser evaluados en cada región del ventrículo estudiado (strain regional) y el promedio de estos valores representa el strain global, lo que refleja la función ventricular global ${ }^{37,38}$.

El strain longitudinal, mide la deformación en el sentido base-ápex de la cavidad, es decir, el acortamiento de la cámara. Como la longitud final de la cavidad (sistólica) es menor que la longitud inicial (diastólica), el porcentaje de la deformación es negativo ${ }^{16}$.

El strain longitudinal global medido por 2D-STE proporciona la oportunidad de cuantificar tanto la magnitud como el tiempo de deformación regional, sistólico y diastólico. El strain longitudinal global se considera como un parámetro eficaz para la cuantificación de la función ventricular izquierda, más sensible que la FEVI por ecocardiografía bidimensional $^{39}$.

Recientemente se publicó un metaanálisis ${ }^{40}$ de 24 estudios que reportó que los valores normales para strain longitudinal global oscilaron entre -15,9\% a $-22,1 \%$ (promedio, -19,7\%; IC 95\%: $-18,9 \%$ a $-20,4 \%)$. Asimismo, investigadores del estudio Framingham reportaron los valores de referencia por sexo del strain longitudinal global en adultos sanos sin evidencia de enfermedades 


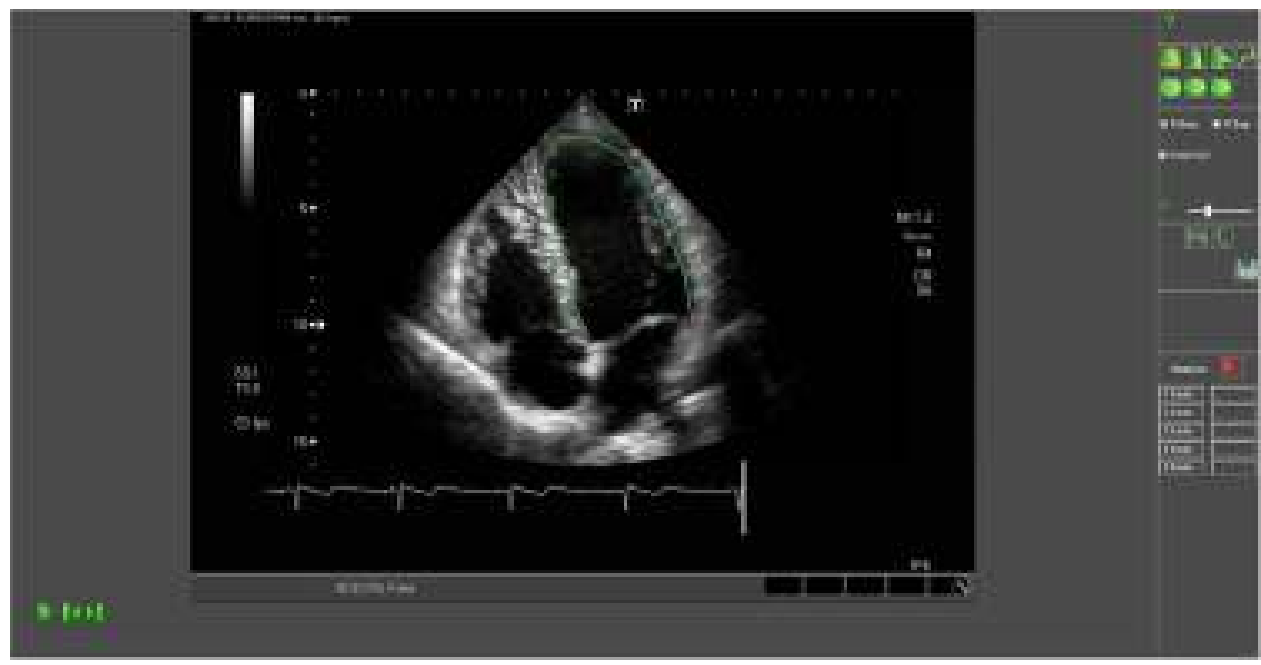

Figura 1 Demarcación de 8 a 12 puntos sobre el borde endocárdico del ventrículo izquierdo por metodología speckle-tracking.

cardiovasculares. Los hallazgos mostraron que el strain longitudinal global fue de $-14,4 \%$ a $-17,1 \%$ en mujeres y $-14,4 \%$ a $-15,2 \%$ en hombres ${ }^{15}$.

Los valores de referencia para strain longitudinal global pueden variar por la disponibilidad de diferentes equipos de imagen ecocardiográfica, así como distintas aplicaciones de software para el análisis de 2D-STE ${ }^{35}$.

\section{Strain longitudinal global y síndrome metabólico}

Investigadores de Francia y Australia señalaron que la identificación temprana de disincronía del miocardio del ventrículo izquierdo por $2 \mathrm{D}-\mathrm{STE}$ en una población de alto riesgo, pero sin síntomas de enfermedades cardiovasculares (pacientes con síndrome metabólico), es importante por varias razones. En primer lugar, esta metodología proporciona un recurso adicional y más sensible para la detección de disfunción sistólica subclínica. En segunda instancia, la disincronía del ventrículo izquierdo también puede permitir la detección precoz de disfunción sistólica aislada. En este estudio, un porcentaje significativo de individuos con síndrome metabólico y sin función diastólica perjudicada, presentaron disincronía del ventrículo izquierdo manifestada por 2D-STE. Se identificó una fuerte interrelación entre disincronía del ventrículo izquierdo, inflamación sistémica y obesidad abdominal en participantes con síndrome metabólico, independientemente del estado diabético. Este resultado puede ayudar a comprender mejor la mecánica del miocardio en las primeras fases de los trastornos metabólicos ${ }^{41}$.

En el mismo sentido, Crendal et $a l^{19}$ en un estudio transversal con 92 adultos con síndrome metabólico y 50 controles sanos, encontraron que los participantes con síndrome metabólico presentaron función miocárdica sistólica y diastólica perjudicada evaluada mediante strain

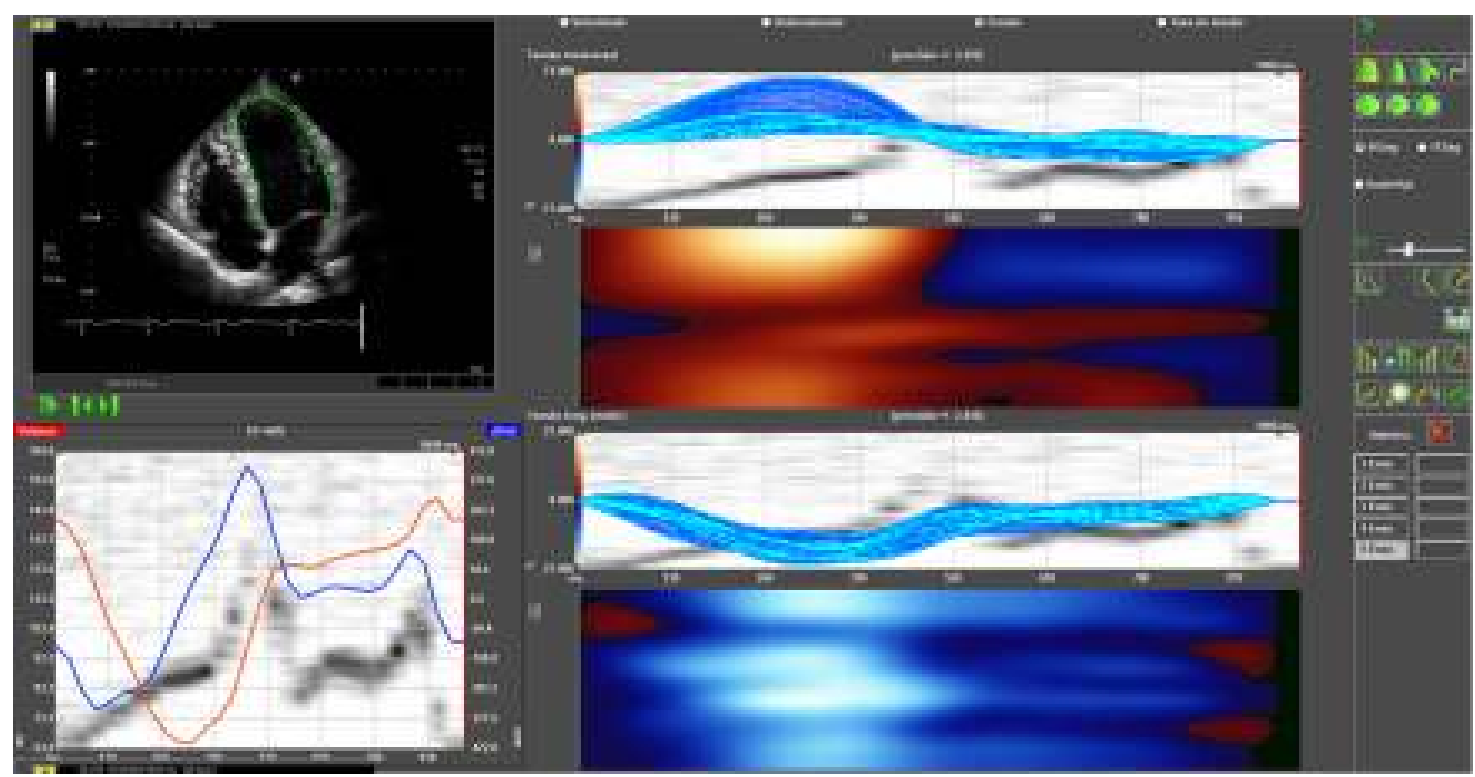

Figura 2 Representación paramétrica del strain longitudinal global. 


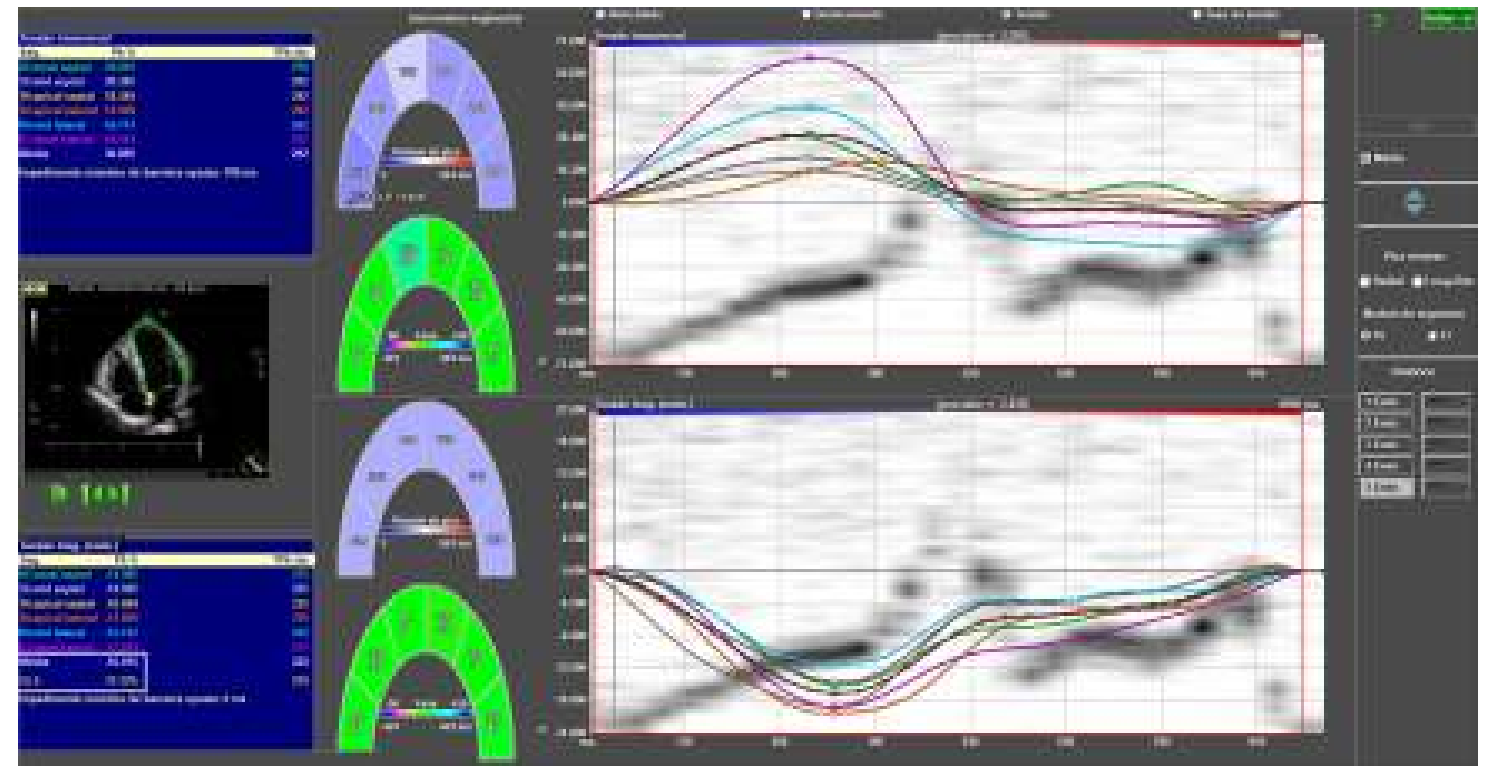

Figura 3 Análisis de strain longitudinal global por segmentos.

longitudinal global. Los resultados también mostraron que la obesidad abdominal, combinada con biomarcadores inflamatorios y marcadores de disincronía sistólica, se asociaron fuertemente con el deterioro de la función miocárdica. Los participantes con diabetes mellitus y mayor número de factores de riesgo metabólicos tenían disfunción miocárdica más grave. Los investigadores también analizaron separadamente los pacientes que presentaron síndrome metabólico y diabetes mellitus versus síndrome metabólico sin diabetes mellitus. Se encontró que el strain longitudinal global fue peor en el grupo de pacientes con síndrome metabólico y diabetes mellitus concomitante ${ }^{41}$.

En el Multi-Ethnic Study of Atherosclerosis-MESA- llevado a cabo en Baltimore - Estados Unidos ${ }^{18}$, en el que se incluyeron 133 participantes, la prevalencia de síndrome metabólico fue del $31 \%$. Los investigadores plantearon que este síndrome se asocia con deterioro de la función miocárdica, evaluada por 2D-STE. Los hallazgos mostraron que los individuos con síndrome metabólico tuvieron valores menores de strain longitudinal global en comparación con aquellos sin síndrome metabólico. En el análisis multivariado se encontró que la presencia de síndrome metabólico ( $\beta=1,3 \%$; IC95\%: 0,3 - 2,2; $p<0,01)$ y la masa del ventrículo izquierdo ( $\beta=0,02 \%$; IC95\%: $0,01-0,03 ; p=0,02)$ se asociaron con un menor valor de strain longitudinal global después de ajustar por etnia, FEVI y creatinina. Los autores concluyeron que el strain longitudinal global del ventrículo izquierdo, es un marcador de enfermedades cardiovasculares subclínicas y está comprometido en individuos asintomáticos con síndrome metabólico y sin historia previa de infarto del miocardio, insuficiencia cardiaca y/o FEVI $<50 \%$.

En otro estudio, investigadores serbios evaluaron la mecánica ventricular izquierda estimada por 2D-STE en 95 pacientes con síndrome metabólico y 65 controles sanos. El strain longitudinal global fue significativamente menor en el grupo de pacientes con síndrome metabólico. El análisis multivariado de los criterios de síndrome metabólico mostró que la presión arterial media en $24 \mathrm{~h}$, la circunferencia de la cintura y la glicemia en ayunas se asociaron independientemente con el strain longitudinal global. Los resultados también mostraron que la interacción entre el sexo y el síndrome metabólico afecta significativamente el strain longitudinal global del ventrículo izquierdo ${ }^{17}$.

En la tabla 1 se observa que el strain longitudinal global en pacientes con síndrome metabólico es significativamente menor cuando se compara con participantes controles o sin síndrome metabólico.

\section{Disfunción ventricular izquierda subclínica, strain longitudinal global y trastornos metabólicos}

Los pacientes con trastornos metabólicos a menudo tienen evidencia de disfunción sistólica y diastólica subclínica por TDI, aun teniendo FEVI normal ${ }^{42}$. En contraste con la FEVI, el strain longitudinal global medido por 2D-STE es más sensible para la detección de disfunción ventricular izquierda subclínica. Esta disfunción es común, incluso en pacientes asintomáticos ${ }^{43}$. Es importante destacar que la técnica por imagen de strain es sensible para identificar disfunción y fibrosis miocárdica asociada con disfunción ventricular izquierda en pacientes con diabetes mellitus tipo $2^{44}$. Datos recientes sugieren que dicha disfunción está presente en casi la mitad de pacientes con diabetes mellitus tipo $2^{45}$. En este sentido, investigadores australianos realizaron un estudio de cohorte prospectivo en 230 participantes, con el objetivo de evaluar el resultado a largo plazo de disfunción ventricular izquierda subclínica asociada con diabetes mellitus tipo 2, durante 10 años de seguimiento. Los hallazgos mostraron que $45 \%$ de la cohorte presentó evidencia de disfunción ventricular izquierda detectada mediante strain longitudinal global. Los autores concluyeron que la disfunción ventricular izquierda subclínica es común en pacientes asintomáticos con diabetes mellitus tipo 2 , fácilmente detectable por imágenes de strain longitudinal 
Tabla 1 Strain longitudinal global en pacientes con síndrome metabólico versus participantes controles

\begin{tabular}{llllll}
\hline Estudio & Año & $n$ & $\begin{array}{l}\text { SLG pacientes con } \\
\text { síndrome metabólico } \\
\text { promedio } \pm \text { DE }\end{array}$ & $\begin{array}{l}\text { SLG } \\
\text { Controles } \\
\text { promedio } \pm \text { DE }\end{array}$ & $\begin{array}{l}\text { Valor de } \\
p\end{array}$ \\
\hline Tadic et al $^{17}$ & 2014 & 160 & $-18,6 \pm 1,6$ & $-21,1 \pm 2,0$ & $<0,001$ \\
Almeida et $_{\text {al }}{ }^{18}$ & 2014 & 133 & $-12,1 \pm 2,5$ & $-13,9 \pm 2,3$ & $<0,01$ \\
Crendal et $_{\text {al }}{ }^{19}$ & 2013 & 108 & $-16,8 \pm 2,8$ & $-21,2 \pm 2,6$ & $<0,001$ \\
\hline
\end{tabular}

DE: desviación estándar; SLG: strain longitudinal global.

global y ésta se asocia de forma independiente con eventos adversos $^{46}$.

A pesar de su papel en la evaluación fundamental de la función sistólica, la FEVI es menos sensible para la detección de enfermedad miocárdica temprana que nuevas técnicas ecocardiográficas como el strain longitudinal global medido por 2D-STE ${ }^{47}$. Recientemente se publicó un estudio americano con resultados de 678 participantes de una cohorte multiétnica de base poblacional. Los investigadores encontraron que el strain longitudinal global fue significativamente más bajo en el grupo de participantes de raza negra $(-16,5 \% \pm 3,5 \%)$ que en los blancos $(-17,5 \% \pm 3,0 \%)$ e hispanos $(-17,3 \% \pm 2,9 \%)$, mientras que la FEVI no fue significativamente diferente en los tres grupos. Adicionalmente, el estudio reportó que en el análisis multivariado ajustado por variables de confusión y factores de riesgo cardiovascular, los individuos de raza negra fueron significativamente más propensos a tener disfunción sistólica del ventrículo izquierdo evaluada con strain longitudinal global $(O R=2,6$, IC95\%: 1,4-4,7) en comparación con los otros grupos ${ }^{48}$.

Se considera que la agrupación de factores de riesgo para enfermedades cardiovasculares que tipifica el síndrome metabólico, será la fuerza de conducción de una nueva epidemia de enfermedades cardiovasculares. Debido a su aumento concomitante en el riesgo de diabetes mellitus y enfermedades cardiovasculares, el síndrome metabólico tendrá una enorme carga sobre las economías del mundo y en la salud pública, de no hacer intervenciones precoces y eficaces ${ }^{22-24}$. La detección y el manejo precoz de enfermedades cardiovasculares subclínicas puede mitigar algunos de los riesgos cardiovasculares asociados con trastornos metabólicos. Con la capacidad de detectar disfunción ventricular izquierda subclínica en pacientes con trastornos metabólicos, el strain longitudinal global por 2D-STE tiene el potencial de convertirse en una herramienta útil para la estratificación de riesgo en este tipo de población ${ }^{14,49}$.

\section{Responsabilidades éticas}

Protección de personas y animales. Los autores declaran que para esta investigación no se han realizado experimentos en seres humanos ni en animales.

Confidencialidad de los datos. Los autores declaran que en este artículo no aparecen datos de pacientes.

Derecho a la privacidad y consentimiento informado. Los autores declaran que en este artículo no aparecen datos de pacientes.

\section{Conflicto de intereses}

Los autores declaran no tener ningún conflicto de intereses.

\section{Anexo. Material adicional}

Se puede consultar material adicional a este artículo en su versión electrónica disponible en doi:10.1016/j.rccar. 2015.10.008.

\section{Bibliografía}

1. GBD 2013 Mortality and Causes of Death Collaborators. Global, regional, and national age-sex specific all-cause and cause-specific mortality for 240 causes of death, 1990-2013: a systematic analysis for the Global Burden of Disease Study 2013. Lancet. 2015; 385:117-71.

2. Yusuf S, Hawken S, Ounpuu S, Dans T, Avezum A, Lanas F, et al. Effect of potentially modifiable risk factors associated with myocardial infarction in 52 countries (the INTERHEART study): case-control study. Lancet. 2004;364:937-52.

3. Reaven GM. Metabolic syndrome. Pathophysiology and implications for management of cardiovascular disease. Circulation. 2002;106:286-8.

4. Isomaa B. A major health hazard: the metabolic syndrome. Life Sci. 2003;73:2395-411.

5. Mottillo S, Filion KB, Genest J, Joseph L, Pilote L, Poirier P, et al. The metabolic syndrome and cardiovascular risk a systematic review and meta-analysis. J Am Coll Cardiol. 2010;56: 1113-32.

6. Galassi A, Reynolds K, He J. Metabolic syndrome and risk of cardiovascular disease: a meta-analysis. Am J Med. 2006;119:812-9.

7. Wu SH, Liu Z, Ho SC. Metabolic syndrome and all-cause mortality: a meta-analysis of prospective cohort studies. Eur J Epidemiol. 2010;25:375-84.

8. Mahmud A, Almuntaser I, Brown A, King G, Crean P, Feely J. Left ventricular structural and functional changes in the metabolic syndrome. J Cardiometab Syndr. 2009;4:81-8.

9. Masugata H, Senda S, Goda F, Yoshihara Y, Yoshikawa K, Fujita $N$, et al. Left ventricular diastolic dysfunction as assessed by echocardiography in metabolic syndrome. Hypertens Res. 2006;29:897-903.

10. Turhan H, Yasar A, Yagmur J, Kurtoglu E, Yetkin E. The impact of metabolic syndrome on left ventricular function: evaluated by using the index of myocardial performance. Int J Cardiol. 2009;132:382-6.

11. Gong HP, Tan HW, Fang NN, Song T, Li SH, Zhong M, et al. Impaired left ventricular systolic and diastolic function in patients with metabolic syndrome as assessed by strain and strain rate imaging. Diabetes Res Clin Pract. 2009;83:300-7.

12. Seo JM, Park TH, Lee DY, Cho YR, Baek HK, Park JS, et al. Subclinical myocardial dysfunction in metabolic syndrome patients 
without hypertension. J Cardiovasc Ultrasound. 2011;19: 134-9.

13. Sengupta PP, Korinek J, Belohlavek M, Narula J, Vannan MA, Jahangir A, et al. Left ventricular structure and function: basic science for cardiac imaging. J Am Coll Cardiol. 2006;48:1988-2001.

14. Shah AM, Solomon SD. Myocardial deformation imaging: current status and future directions. Circulation. 2012;125:e244-8.

15. Cheng S, Larson MG, McCabe EL, Osypiuk E, Lehman BT, Stanchev $P$, et al. Age- and sex-based reference limits and clinical correlates of myocardial strain and synchrony: the Framingham Heart Study. Circ Cardiovasc Imaging. 2013;6:692-9.

16. Del Castillo JM, Herszkowicz N, Ferreira C. Speckle tracking a contratilidade miocárdica em sintonia fina. Rev Bras Ecocardiogr Imagem Cardiovasc. 2010;23:46-54.

17. Tadic M, Cuspidi C, Majstorovic A, Pencic B, Backovic S, Ivanovic $B$, et al. Does the metabolic syndrome impact left-ventricular mechanics? A two-dimensional speckle tracking study. J Hypertens. 2014;32:1870-8.

18. Almeida AL, Teixido-Tura G, Choi EY, Opdahl A, Fernandes VR, Wu CO, et al. Metabolic syndrome, strain, and reduced myocardial function: multi-ethnic study of atherosclerosis. Arq Bras Cardiol. 2014;102:327-35.

19. Crendal E, Walther G, Vinet A, Dutheil F, Naughton G, Lesourd $B$, et al. Myocardial deformation and twist mechanics in adults with metabolic syndrome: impact of cumulative metabolic burden. Obesity (Silver Spring). 2013;21:e679-86.

20. Almeida ALC, Gjesdal O, Mewton N, Young Choi E, Teixido-Tura G, Yoneyama K, et al. Speckle-tracking pela ecocardiografia bidimensional - aplicações clínicas. Rev Bras Ecocardiogr Imagem Cardiovasc. 2013;26:38-49.

21. St Vrain J, Bilhorn K, Kurup S, Peterson LR. Strain imaging using speckle tracking in the cardiometabolic syndrome: method and utility. J Cardiometab Syndr. 2008;3:258-61.

22. Gami AS, Witt BJ, Howard DE, Erwin PJ, Gami LA, Somers VK, et al. Metabolic syndrome and risk of incident cardiovascular events and death: a systematic review and meta-analysis of longitudinal studies. J Am Coll Cardiol. 2007;49:403-14.

23. Stern M, Williams K, Gonzalez-Villalpando C, Hunt KJ, Haffner $S M$. Does the metabolic síndrome improve identification of individuals at risk of type 2 diabetes and/or cardiovascular disease? Diabetes Care. 2004;27:2676-81.

24. Ford ES, Li C, Sattar N. Metabolic syndrome and incident diabetes: current state of evidence. Diabetes Care. 2008;31:1898-904.

25. Grundy SM. Metabolic syndrome pandemic. Arterioscler Thromb Vasc Biol. 2008;28:629-36.

26. Reynolds K, He J. Epidemiology of the metabolic syndrome. Am J Med Sci. 2005;330:273-9.

27. Hossain P, Kawar B, El Nahas M. Obesity and diabetes in the developing world-a growing challenge. N Engl J Med. 2007;356:213-5.

28. Palaniappan L, Carnethon MR, Wang Y, Hanley AJ, Fortmann SP, Haffner SM, et al. Predictors of the incident metabolic syndrome in adults: the Insulin Resistance Atherosclerosis Study. Diabetes Care. 2004;27:788-93.

29. Wilsgaard T, Jacobsen BK. Lifestyle factors and incident metabolic syndrome. The Tromsø Study 1979-2001. Diabetes Res Clin Pract. 2007;78:217-24

30. Cheung BM, Wat NM, Tam S, Thomas GN, Leung GM, Cheng CH, et al. Components of the metabolic syndrome predictive of its development: a 6-year longitudinal study in Hong Kong Chinese. Clin Endocrinol (Oxf). 2008;68:730-7.

31. Sheu WH, Chuang SY, Lee WJ, Tsai ST, Chou P, Chen CH. Predictors of incident diabetes, metabolic syndrome in middle-aged adults: a 10-year follow-up study from Kinmen, Taiwan. Diabetes Res Clin Pract. 2006;74:162-8.
32. Day C. Metabolic syndrome, or what you will: definitions and epidemiology. Diab Vasc Dis Res. 2007;4:32-8.

33. Alberti KG, Eckel RH, Grundy SM, Zimmet PZ, Cleeman JI, Donato KA, et al. Harmonizing the Metabolic Syndrome. A Joint Interim Statement of the International Diabetes Federation Task Force on Epidemiology and Prevention; National Heart, Lung, and Blood Institute; American Heart Association; World Heart Federation; International Atherosclerosis Society; and International Association for the Study of Obesity. Circulation. 2009;120:640-5.

34. Mondillo S, Galderisi M, Mele D, Cameli M, Lomoriello VS, Zacà V, et al. Speckle-tracking echocardiography: a new technique for assessing myocardial function. J Ultrasound Med. 2011;30:71-83

35. Voigt JU, Pedrizzetti G, Lysyansky P, Marwick TH, Houle H, Baumann R, et al. Definitions for a common standard for 2D speckle tracking echocardiography: consensus document of the EACVI/ASE/Industry Task Force to standardize deformation imaging. J Am Soc Echocardiogr. 2015;28:183-93.

36. Urheim S, Edvardsen T, Torp H, Angelsen B, Smiseth OA. Myocardial strain by doppler echocardiography. Validation of a new method to quantify regional myocardial function. Circulation. 2000;102:1158-64.

37. Reisner SA, Lysyansky P, Agmon Y, Mutlak D, Lessick J, Friedman Z. Global longitudinal strain: a novel index of left ventricular systolic function. J Am Soc Echocardiogr. 2004;17: 630-3.

38. D’Hooge J, Heimdal A, Jamal F, Kukulski T, Bijnens B, Rademakers $\mathrm{F}$, et al. Regional strain and strain rate measurements by cardiac ultrasound: Principles, implementation and limitations. Eur J Echocardiogr. 2000;1:154-70.

39. Cimino S, Canali E, Petronilli V, Cicogna F, De Luca L, Francone $M$, et al. Global and regional longitudinal strain assessed by twodimensional speckle tracking echocardiography identifies early myocardial dysfunction and transmural extent of myocardial scar in patients with acute ST elevation myocardial infarction and relatively preserved LV function. Eur Heart J Cardiovasc Imaging. 2013;14:805-11.

40. Yingchoncharoen T, Agarwal S, Popović ZB, Marwick TH. Normal ranges of left ventricular strain: a meta-analysis. J Am Soc Echocardiogr. 2013;26:185-91.

41. Crendal E, Walther G, Dutheil F, Courteix D, Lesourd B, Chapier $\mathrm{R}$, et al. Left ventricular myocardial dyssynchrony is already present in nondiabetic patients with metabolic syndrome. Can J Cardiol. 2014;30:320-4.

42. Vinereanu D, Nicolaides E, Tweddel AC, Mädler CF, Holst B, Boden LE, et al. Subclinical left ventricular dysfunction in asymptomatic patients with Type II diabetes mellitus, related to serumlipids and glycated haemoglobin. Clin Sci (Lond). 2003;105:591-9.

43. Ng AC, Delgado V, Bertini M, van der Meer RW, Rijzewijk LJ, Shanks $M$, et al. Findings from left ventricular strain and strain rate imaging in asymptomatic patients with type 2 diabetes mellitus. Am J Cardiol. 2009;104:1398-401.

44. Saito M, Okayama H, Yoshii T, Higashi H, Morioka H, Hiasa G, et al. Clinical significance of global two-dimensional strain as a surrogate parameter of myocardial fibrosis and cardiac events in patients with hypertrophic cardiomyopathy. Eur Heart J Cardiovasc Imaging. 2012;13:617-23.

45. Faden G, Faganello G, De Feo S, Berlinghieri N, Tarantini L, Di Lenarda A, et al. The increasing detection of asymptomatic left ventricular dysfunction in patients with type 2 diabetes mellitus without overt cardiac disease: data from the SHORTWAVE study. Diabetes Res Clin Pract. 2013;101:309-16.

46. Holland DJ, Marwick TH, Haluska BA, Leano R, Hordern MD, Hare $\mathrm{JL}$, et al. Subclinical LV dysfunction and 10-year outcomes in type 2 diabetes mellitus. Heart. 2015;101:1061-6. 
47. Stanton T, Leano R, Marwick TH. Prediction of all-cause mortality from global longitudinal speckle strain: comparison with ejection fraction and wall motion scoring. Circ Cardiovasc Imaging. 2009;2:356-64.

48. Russo C, Jin Z, Homma S, Rundek T, Elkind MS, Sacco RL, et al. Race-ethnic differences in subclinical left ventricular systolic dysfunction by global longitudinal strain: a community-based cohort study. Am Heart J. 2015;169:721-6.

49. Fitchett D, Connelly K. Impaired cardiac function in metabolic syndrome. Can J Cardiol. 2014;30:270-1. 